\title{
Atomistic simulations of wetting properties and water films on hydrophilic surfaces
}

Matej Kanduč and Roland R. Netz

Citation: The Journal of Chemical Physics 146, 164705 (2017); doi: 10.1063/1.4979847

View online: http://dx.doi.org/10.1063/1.4979847

View Table of Contents: http://aip.scitation.org/toc/jcp/146/16

Published by the American Institute of Physics

\section{Articles you may be interested in}

Molecular dynamics simulation of nanobubble nucleation on rough surfaces

The Journal of Chemical Physics 146, 164704 (2017); 10.1063/1.4981788

Wetting at the nanoscale: A molecular dynamics study

The Journal of Chemical Physics 146, 114704 (2017); 10.1063/1.4978497

Perspective: Dissipative particle dynamics

The Journal of Chemical Physics 146, 150901 (2017); 10.1063/1.4979514

A Gaussian theory for fluctuations in simple liquids

The Journal of Chemical Physics 146, 134507 (2017); 10.1063/1.4979659

Communication: Slow proton-charge diffusion in nanoconfined water

The Journal of Chemical Physics 146, 131101 (2017); 10.1063/1.4979714

Rotation driven translational diffusion of polyatomic ions in water: A novel mechanism for breakdown of Stokes-Einstein relation

The Journal of Chemical Physics 146, 164502 (2017); 10.1063/1.4981257

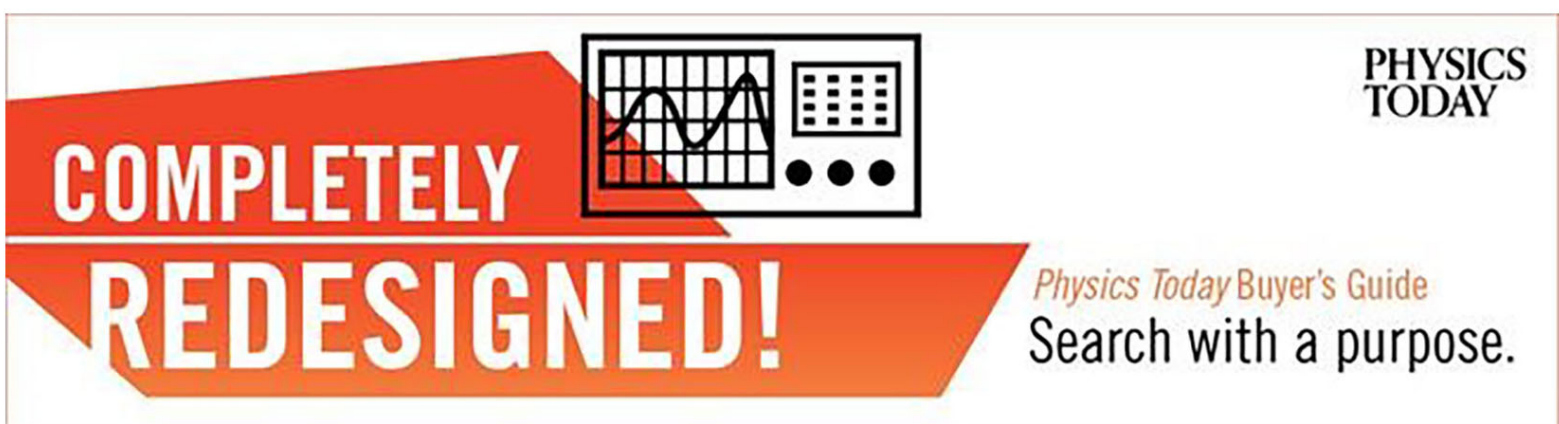




\title{
Atomistic simulations of wetting properties and water films on hydrophilic surfaces
}

\author{
Matej Kanduč ${ }^{1, a)}$ and Roland R. Netz ${ }^{2}$ \\ ${ }^{1}$ Soft Matter and Functional Materials, Helmholtz-Zentrum Berlin für Materialien und Energie, \\ Hahn-Meitner-Platz 1, D-14109 Berlin, Germany \\ ${ }^{2}$ Fachbereich Physik, Freie Universität Berlin, Arnimallee 14, D-14195 Berlin, Germany
}

(Received 25 January 2017; accepted 20 March 2017; published online 24 April 2017)

\begin{abstract}
We use molecular simulations to investigate the wetting behavior of water at flat polar surfaces. Introducing a computational procedure based on thermodynamic integration methods, we determine the equilibrium water film thickness on the surface at given vapor density as well as the corresponding change of the surface free energy. The wetting film is relevant on polar surfaces near the wetting transition and significantly alters the surface contact angle. For thin films, the surface free energy change increases linearly with the thickness, as predicted by simple thermodynamic arguments. For thick films we observe deviations from linearity, which we rationalize by the formation of hydrogen bonds between water molecules in the film. Our approach provides an efficient and accurate technique to calculate the wetting properties of surface layers, which we verify by simulating water droplets on the surfaces. Published by AIP Publishing. [http://dx.doi.org/10.1063/1.4979847]
\end{abstract}

\section{INTRODUCTION}

Wetting represents one of the most fundamental features of water interaction with a surface, resulting from interplay between cohesive and adhesive molecular forces. Wetting properties play a paramount role in many scientific and technological fields, ranging from nanofluidics and microfluidics to nanoprinting. ${ }^{1-3}$ Water also represents a common operating liquid in many industrial processes on larger scales such as dyeing, painting, coating, lubrication, oil recovery, and deposition of pesticides. ${ }^{4}$ Therefore, there is considerable interest in a better understanding how water behaves near solid surfaces.

The wetting properties of a liquid depend on the surface chemistry, which dictates the strength of the solid-liquid interaction, and the surface structure, including the nanoscale topography and the atomistic-scale organization of atoms within the substrate. The most direct experimental approach to quantify the interaction between a surface and a liquid is by measuring the contact angle a macroscopic drop forms on that surface. From the thermodynamic viewpoint, the equilibrium contact angle formed by a liquid droplet on a solid is related to the surface tensions of the system, which can be expressed via the wetting coefficient $k_{\mathrm{w}}$ as ${ }^{5}$

$$
k_{\mathrm{w}}=\frac{\gamma_{\mathrm{sv}}-\gamma_{\mathrm{sw}}}{\gamma} .
$$

Here, $\gamma_{\mathrm{sv}}, \gamma_{\mathrm{sw}}$, and $\gamma$ stand for surface-vapor, surface-water, and water-vapor surface tensions, respectively. The wetting coefficient reflects the water binding affinity to the surface and is via Young's equation related to the contact angle as

$$
\cos \theta=k_{\mathrm{w}} .
$$

\footnotetext{
a)matej.kanduc@helmholtz-berlin.de
}

If a surface is in contact with a water vapor, a water film forms on the surface. A surface exposed to atmospheric conditions is therefore never completely dry. The presence of such a water film influences surface properties and if its thickness is significant, it modifies the surface-vapor surface tension. ${ }^{1,6}$ Apart from the surface type, the thickness of the film depends on the vapor density $\rho_{\mathrm{v}}$. Therefore, the surface-vapor surface tension is generally a function of the vapor density, $\gamma_{\mathrm{sv}}\left(\rho_{\mathrm{v}}\right)$. In the case where a surface is placed in vacuum or in a completely dry atmosphere, no film is present and one speaks of the surfacevacuum surface tension or simply the surface tension of a dry surface, $\gamma_{\mathrm{s}} \equiv \gamma_{\mathrm{sv}}\left(\rho_{\mathrm{v}} \rightarrow 0\right){ }^{7}$ Therefore, one distinguishes between "dry" and "moist" surface tensions, depending on whether the surface is in vacuum or in equilibrium with a vapor phase. ${ }^{1,6,8}$

Theoretical fundamentals of wetting have been reviewed by de Gennes. ${ }^{6}$ In recent years, our understanding of the molecular mechanisms that drive the interfacial thermodynamics of solid-liquid interfaces has been advanced by molecular simulations in combination with concepts of statistical mechanics. ${ }^{9-15}$ Several advanced computational techniques have been developed that enable the computation of a surface-water adhesion free energy. ${ }^{16-19}$ Usually, these techniques neglect the formation of a water film, which is a justified assumption for not too hydrophilic surfaces. However, wetting becomes important for situations close to the wetting transition. Errington and co-workers have introduced a Monte Carlo method that provides the surface excess free energy associated with a liquid film on a surface. ${ }^{8,20-23}$ The method is suitable for deducing the wetting properties of systems within the partial wetting regime, such as the wetting coefficient, the contact angle, and the thickness of the adsorbed liquid film. It has been also used to analyze the wetting behavior of water near flat non-polar surfaces. ${ }^{23}$ Despite the progress in computer modeling, the understanding of the influence of a finite 
water film on the wetting properties on polar surfaces is still insufficient.

In this work, we employ an atomistic model of a surface with tunable polarity, ranging from a hydrophobic to a very hydrophilic surface. This enables us to systematically study wetting on a wide range of surface polarities in the presence of water vapor and its influence on the surface free energy. To that end, we introduce and describe in detail in Section II a thermodynamic integration (TI) procedure suitable for Molecular Dynamics (MD) simulations, which enables accurate determination of surface wetting coefficients. The method uses standard routines implemented in existing MD packages and does not need code modifications. The wetting free energy obtained for a given relative vapor humidity can be trivially transformed to the situation of varying humidity, which offers a general description of wetting and wetting phenomena. The observed relation between the thickness of the adsorbed film and the change in the surface wetting coefficient is explained by simple thermodynamic arguments in Section III. We verify the TI results by independent simulations of a water droplet on the surface.

\section{METHODS}

\section{A. Model}

We set up a simple atomistic model already introduced in our previous studies on hydration interactions between surfaces: ${ }^{18,24}$ a planar surface, modeling a self-assembled monolayer, is composed of ten-carbon-atom long alkane chains terminated by polar hydroxyl $(-\mathrm{OH})$ head groups with adjustable polarity, see Fig. 1. The molecules are arranged on a hexagonal lattice with area density of $4.3 \mathrm{~nm}^{-2}$ and stabilized by restraining the second carbon atom from the head group by harmonic potentials of strength $k_{x}=k_{y}=500(\mathrm{~kJ} / \mathrm{mol}) / \mathrm{nm}^{2}$ in lateral directions and $k_{z}=10(\mathrm{~kJ} / \mathrm{mol}) / \mathrm{nm}^{2}$ in the normal direction. The polarity of the surface (and thereby its contact angle) is tuned by scaling the partial charges of the head groups by a factor $\alpha$ in the range between 0 and 1. For $\alpha=0$, the surface is completely non-polar, with head groups resembling methylated termini, whereas for $\alpha=1$, it is completely

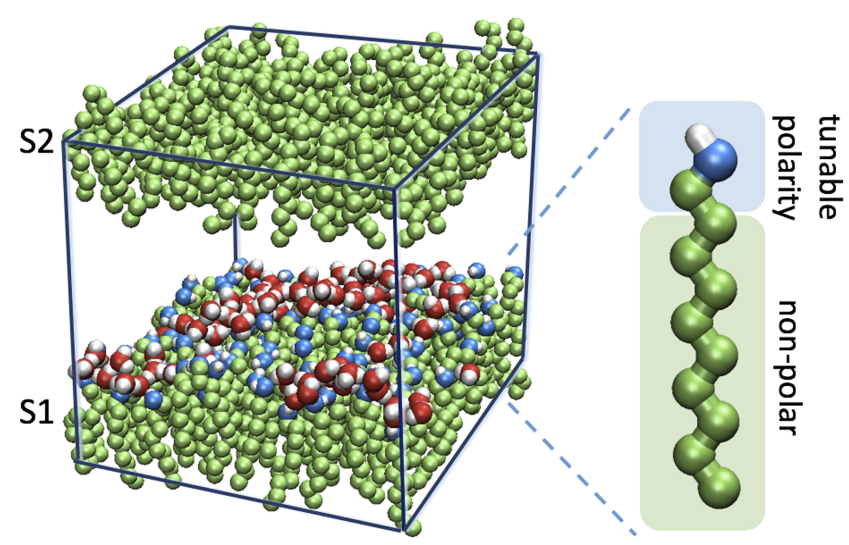

FIG. 1. Setup of the system composed of a wet surface (S1), which is the surface of interest, and a confining surface (S2). Each surface molecule consists of an alkane chain terminated by a modified hydroxyl group whose polarity is rescaled by a polarity factor $\alpha$. A thin water film (in this case comprised of 100 water molecules) is adsorbed on surface S1. Simulation box (blue frame) is replicated in all three directions via periodic boundary conditions. polar with head groups that mimic hydroxyl head groups. In order to prevent the head groups to form hydrogen bonds (HBs) with themselves, ${ }^{15}$ we increase the repulsive coefficient in the Lennard-Jones (LJ) interaction between oxygen atoms in the $\mathrm{OH}$ groups to $C_{12}=10^{5} \mathrm{~nm}^{12} \mathrm{~kJ} \mathrm{~mol}^{-1}$ such that the LJ diameter is $\sigma_{\mathrm{LJ}}=0.4 \mathrm{~nm}$. A thorough discussion on the implications of intra-surface hydrogen bonding can be found in Ref. 15 .

In the simulations used for the evaluation of the wetting free energy, the lateral box size is $4.5 \mathrm{~nm} \times 5.2 \mathrm{~nm}$ replicated in all three directions via periodic boundary conditions. In the simulations with water droplets, we use larger box dimensions as explained separately.

Parallel to the first (wet) surface (S1), which is the surface of interest, we place a confining surface (S2), which can be either identical to the first one (for evaluating the wetting coefficient), or non-adsorbing, i.e., very hydrophobic (for studying the water film), as we will explain later. In the slit region between both surfaces, we insert $N$ water molecules.

We use united-atom parameters taken from the GROMOS force field for the chains ${ }^{25}$ and the SPC/E water model..$^{26}$ The MD simulations are performed with the GROMACS simulation package $\mathrm{e}^{27,28}$ in the canonical isochoric ensemble (NVT) at $T=300 \mathrm{~K}$ maintained by the Berendsen thermostat with a time constant of $1 \mathrm{ps}^{29}$ and an integration time step of $2 \mathrm{fs}$. A plain cutoff of $0.9 \mathrm{~nm}$ is used for short-range LJ interactions. Electrostatics is treated using Particle-Mesh-Ewald (PME) methods ${ }^{30,31}$ with a $0.9 \mathrm{~nm}$ real-space cutoff.

\section{B. Dry wetting coefficients}

The dry wetting coefficient, related to a surface in a dry atmosphere or in vacuum, $k_{\mathrm{w}}^{\mathrm{vac}}$, is associated with the free energy of complete cavitation, that is, the work needed to expel all the water from a fully hydrated slit between two identical surfaces (of type S1) far apart into an external reservoir, as schematically depicted in Fig. 2. The cavitation free energy $F_{\text {cav }}$ can be expressed in terms of the surface tensions as ${ }^{32}$

$$
\begin{aligned}
F_{\text {cav }} & =2\left(\gamma_{\mathrm{s}}-\gamma_{\mathrm{sw}}\right) A \\
& =2 \gamma k_{\mathrm{w}}^{\mathrm{vac}} A,
\end{aligned}
$$

where $\gamma_{\mathrm{s}}$ is the surface tension of the surface in vacuum and $\gamma_{\mathrm{sw}}$ the surface tension in water, $A$ is the surface area, and the prefactor 2 comes from the fact that we have two identical surfaces. We compute the cavitation free energy $F_{\text {cav }}$ via the Thermodynamic Integration (TI) technique as modified by us in Ref. 24. We provide a quick summary of the method, since a similar procedure will be used to evaluate the interface potentials in Sec. II C.

We start with a system of two identical parallel surfaces separated by a 3-nm-thick water phase such that both surfaces do not interact with each other (state A in Fig. 2). The normal pressure (in the $z$ direction) is set to 1 bar, which corresponds to the reference chemical potential $\mu_{0}$ of bulk liquid water at atmospheric conditions. At the same time, we assume the surface to be in contact with saturated vapor of density $\rho_{\mathrm{v}}^{\text {sat }}$. This approximation is justified since the difference between liquid water properties at $1 \mathrm{bar}$ and at the much lower liquid-vapor coexistence pressure are numerically negligible. Treating the vapor as an ideal gas, the reference chemical potential $\mu_{0}$ can be expressed as 

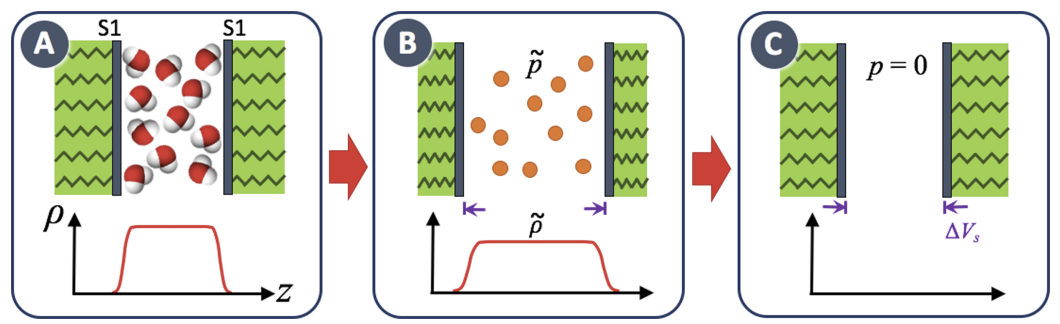

$$
k_{\mathrm{B}} T \log \rho_{\mathrm{v}}^{\mathrm{sat}}=k_{\mathrm{B}} T \log \rho_{\mathrm{w}}+\mu_{\mathrm{w}}^{\mathrm{ex}} \equiv \mu_{0} .
$$

Here, $\rho_{\mathrm{w}}=33.0 \mathrm{~nm}^{-3}$ is the density and $\mu_{\mathrm{w}}^{\mathrm{ex}}=-29.03 \mathrm{~kJ} / \mathrm{mol}$ the excess chemical potential of bulk liquid SPC/E water at $300 \mathrm{~K}^{15,24}$ This leads to the value for the saturated vapor density $\rho_{\mathrm{v}}^{\text {sat }}=2.91 \times 10^{-4} \mathrm{~nm}^{-3}$, close to previously reported values for the SPC/E water model. ${ }^{33}$

In the first step $(\mathrm{A} \rightarrow \mathrm{B})$, we perform TI at constant volume by switching off all water partial charges and all $\mathrm{LJ}$ interactions between water molecules and by that transforming the interlamellar liquid water into an ideal gas. Introducing the coupling parameter $\lambda \in[0,1]$ that gradually switches the interactions in the Hamiltonian $U(\lambda)$ between the original water interactions (for $\lambda=0$ ) and an ideal gas (for $\lambda=1$ ), the excess part of interlamellar water is computed as ${ }^{34}$

$$
F_{\mathrm{TI}}=\int_{0}^{1}\left\langle\frac{\partial U(\lambda)}{\partial \lambda}\right\rangle_{\lambda} \mathrm{d} \lambda .
$$

We perform this switching in two stages: we first linearly scale down the partial charges of water molecules, while keeping their LJ interactions intact. In the second stage, we scale the water LJ interactions using the "soft-core" LJ functions of GROMACS ${ }^{27,28}$ in order to circumvent singularities when the potentials are about to vanish ( $\lambda$ close to 1$)$. ${ }^{35}$ We separate the entire TI procedure into 24 individual simulations with different $\lambda$ values for the Coulomb part and 24 simulations for the LJ part.

In state B, we are left with an ideal gas between the surfaces at an elevated pressure $\tilde{p}$, which is typically $\sim 10^{3}$ bars. The density profile of the ideal gas reaches a plateau of a value $\tilde{\rho}$ in the middle, where the gas molecules are not influenced by the potentials from the surfaces. The chemical potential of the ideal gas can then simply be expressed as $\mu=k_{\mathrm{B}} T \log \tilde{\rho}$.

In the next step $(\mathrm{B} \rightarrow \mathrm{C})$, we hypothetically expel the ideal gas into an external reservoir. Transferring $\mathrm{d} N$ molecules from the slit into the reservoir changes the free energy of the slit by $-\mu(N) \mathrm{d} N$, where $\mu(N)$ is the chemical potential of the gas in the slit. At the same time, we insert these $\mathrm{d} N$ molecules into the reservoir and by that increase its free energy by $\mu_{0} \mathrm{~d} N$. Transferring all $N$ molecules into the reservoir requires the work of $-\int_{0}^{N} \mu\left(N^{\prime}\right) \mathrm{d} N^{\prime}+\mu_{0} N$, which after the integration of the first term yields

$$
\Delta W_{\mu}=-N k_{\mathrm{B}} T\left(\log \frac{\tilde{\rho}}{\rho_{\mathrm{v}}^{\mathrm{sat}}}-1\right),
$$

where we expressed the chemical potential of the reservoir $\mu_{0}$ in terms of $\rho_{\mathrm{v}}^{\text {sat }}$ as given by Eq. (4).

Additionally, by expelling the ideal gas into the reservoir, the pressure between the surfaces drops from $\tilde{p}$ to 0 , which releases the elastic energy previously stored in the soft surfaces

$$
\Delta W_{\mathrm{el}}=-\frac{1}{2} \tilde{p} \Delta V_{\mathrm{s}},
$$

where $\Delta V_{\mathrm{s}}$ is the volume change of the surface slabs after the expulsion of the ideal gas. The value of $\Delta V_{\mathrm{s}}$ is obtained by simulating the cavitated system (state $\mathrm{C}$ ) and comparing the volume of the surface slabs to the one in state B.

Summing up all contributions gives the free energy of cavitation

$$
F_{\mathrm{cav}}=F_{\mathrm{TI}}+\Delta W_{\mu}+\Delta W_{\mathrm{el}}
$$

In fact, the first two terms represent the dominating contributions, whereas the elastic term is merely a correction that typically accounts for less than around 0.1 of the final value of the wetting coefficients. The dry wetting coefficient $k_{\mathrm{w}}^{\mathrm{vac}}$ can then be evaluated by using Eq. (3). This method enables a very accurate determination of the wetting coefficient with an uncertainty of $\delta k_{\mathrm{w}}^{\mathrm{vac}}<0.02$.

\section{Interface potentials}

We now describe a variant of the above method, which we use to evaluate the free energy of a liquid film that forms on a surface. The model setup is schematically depicted in Fig. 3 and comprises two different surfaces: the first one, labeled $\mathrm{S} 1$, is the surface on which the wetting film forms, whereas the other surface, $\mathrm{S} 2$, serves solely as a confining
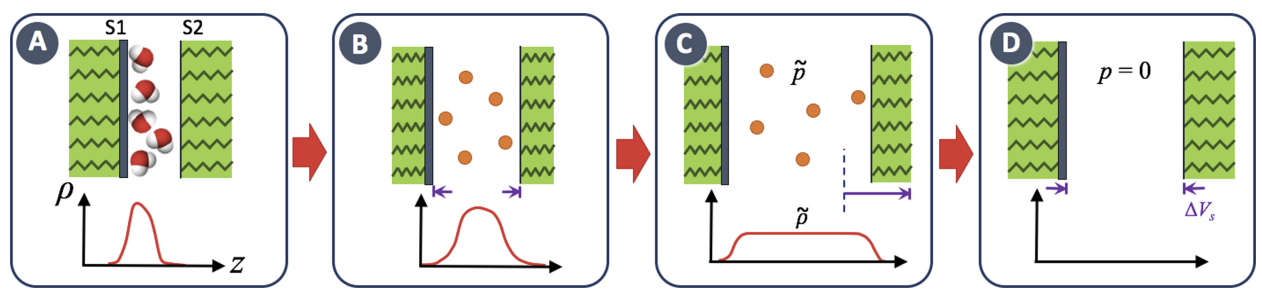

FIG. 3. Thermodynamic route for evaluating the interface potential of a wetting film $\Delta f_{\mathrm{w}}\left(n_{A}\right)$. (A) A film of $N_{\text {ads }}$ water molecules is adsorbed on surface S1. (B) Transforming the film into an ideal gas. (C) By increasing the distance between the surfaces, a plateau with a value $\tilde{\rho}$ forms in the middle of the density profile, which enables the determination of the chemical potential. (D) Upon expelling the gas from the slit, the pressure drops to zero and the surfaces expand by the volume $\Delta V_{\mathrm{s}}$. 
boundary for water molecules. Surface S2 has similar composition as surface $\mathrm{S} 1$ but is characterized by $\alpha=0$ and modified $\mathrm{LJ}$ parameters for the cross interactions between the surface and the water oxygen atoms: $C_{6}=0$ and $C_{12}=9 \times 10^{-5} \mathrm{~nm}^{12}$ $\mathrm{kJ} \mathrm{mol}^{-1}$ such that it exhibits vanishing affinity to water. We insert $N$ water molecules into the system (state A), which predominantly adsorb on surface S1 due to its hydrophilic character.

An important requirement for the application of TI is that the thermodynamic path should be reversible and hysteresis free. Our integration path, although reversible, can cross a firstorder phase transition, since the water-water and Coulombic surface-water interactions are gradually switched off during the TI procedure, which can induce a dewetting transition. It is therefore important to sample the $\lambda$ states at the transition sufficiently long compared with the transition times. In order to minimize the relaxation time, we place surface $S 2$ close to surface S1, yet still far enough not to influence the film, which is adsorbed on surface S1. To that end, the density profiles of the water film and of surface S2 should not overlap. This was not considered in our preliminary study, ${ }^{24}$ which led to slightly different final results of the assessed thicknesses of equilibrium films.

Next, we follow a very similar protocol as for evaluating the cavitation free energy in Section II B. If the surfaces are close to each other, which is the case for thin films, the gas density profile after the first step $(\mathrm{A} \rightarrow \mathrm{B})$ does not reach a plateau value in the middle (as depicted in B). This prevents an easy determination of the gas chemical potential via its density. In the second step, we therefore expand the system in the $z$ direction $(B \rightarrow C)$ such that a plateau appears in the density profile. The chemical potential of the ideal gas in state $\mathrm{C}$ is then $\mu=k_{\mathrm{B}} T \log \tilde{\rho}$, where $\tilde{\rho}$ is the plateau density. In step $\mathrm{B} \rightarrow \mathrm{C}$, the free energy of the system changes by

$$
W_{\exp }=-\int_{\mathrm{B}}^{\mathrm{C}} p(V) \mathrm{d} V .
$$

Here, $p(V)$ is the pressure of the gas at a given simulation box volume $V$, and we integrate from the volume of state $\mathrm{B}$ to the volume of state C. Finally, we hypothetically expel the ideal gas into an external reservoir $(\mathrm{C} \rightarrow \mathrm{D})$, which requires the work given by Eq. (6). As before, the pressure drop from $\tilde{p}$ (in state $\mathrm{C}$ ) to 0 (in state $\mathrm{D}$ ) releases the elastic energy stored in the surfaces, which is given by Eq. (7).

Summing up all the contributions gives us the free energy difference between the surface with the adsorbed film (state A) and the state of a completely dry surface (state D), which we define as the interface potential $\Delta f_{\mathrm{w}}\left(n_{A}\right),{ }^{21,36,37}$

$$
\Delta f_{\mathrm{w}}\left(n_{A}\right)=-\frac{1}{A}\left(F_{\mathrm{TI}}+W_{\mathrm{exp}}+\Delta W_{\mu}+\Delta W_{\mathrm{el}}\right),
$$

where we have expressed the thickness of the water film in terms of the number of adsorbed water molecules $N_{\text {ads }}$ per surface area $A, n_{A}=N_{\text {ads }} / A$. Alternatively, the film amount can be expressed also in terms of an effective thickness as $l=n_{\mathrm{A}} / \rho_{\mathrm{w}}$, which assumes bulk water density $\rho_{\mathrm{w}}=33 \mathrm{~nm}^{-3}$. By definition, $\Delta f_{\mathrm{w}}(0)=0$, and the equilibrium film thickness is found by minimizing $\Delta f_{\mathrm{w}}\left(n_{A}\right)$ over $n_{A}$.

With the above procedure, we can evaluate the wetting coefficient reduction $\Delta k_{\mathrm{w}}$ due to film formation very accurately, with an uncertainty of $\delta k_{\mathrm{w}}<0.01$. The corresponding uncertainty in the contact angle is therefore around $\delta \theta \approx 1^{\circ}$. We apply this method to study the wetting properties of water on polar surfaces, but the method is general and applicable also to other liquids.

\section{RESULTS AND DISCUSSION}

\section{A. Dry wetting coefficients}

We now focus on the water film formation from a surrounding water vapor and its influence on the surface contact angle at fixed temperature of $300 \mathrm{~K}$. The vapor density determines the chemical potential of the system and hence influences the film thickness. In the following, we express the vapor density in terms of the relative humidity $(\mathrm{RH})$, defined as the ratio of the vapor density $\rho_{\mathrm{v}}$ and the saturated vapor density $\rho_{\mathrm{v}}^{\text {sat }}$,

$$
\mathrm{RH}=\frac{\rho_{\mathrm{v}}}{\rho_{\mathrm{v}}^{\text {sat }}} .
$$

We first evaluate the dry wetting coefficients $k_{\mathrm{w}}^{\mathrm{vac}}$, relevant for dry surfaces in vacuum $(\mathrm{RH}=0)$. The latter has been evaluated in our previous studies ${ }^{15,18,24}$ and is plotted in Fig. 4(a) by black squares. Starting with a totally non-polar surface $(\alpha=0)$, the dry wetting coefficient has a value of $k_{\mathrm{w}}^{\mathrm{vac}} \simeq-0.7$,
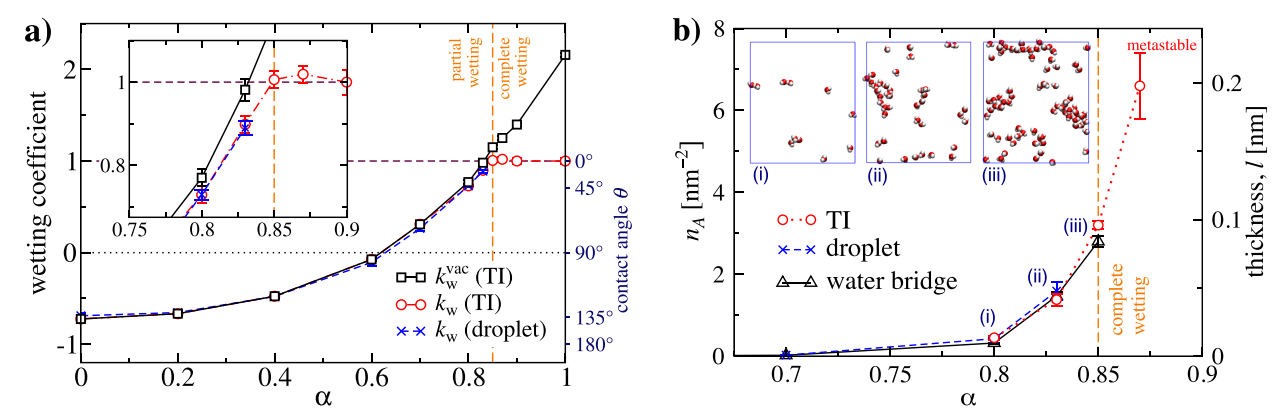

FIG. 4. (a) Wetting coefficient for the surface in vacuum $k_{\mathrm{w}}^{\mathrm{vac}}$ (black squares) and in saturated water vapor $k_{\mathrm{w}}$ (red circles) as a function of the surface polarity $\alpha$. The corresponding contact angle $\theta$ is shown on the right axis. The wetting coefficient $k_{\mathrm{w}}$ obtained from the droplet simulations is shown for comparison by blue crosses. The inset shows an enlarged region near the wetting transition, featuring the difference between $k_{\mathrm{w}}^{\mathrm{vac}}$ and $k_{\mathrm{w}}$ as well as a perfect agreement between the TI and droplet results for $k_{\mathrm{W}}$. (b) Adsorbed film density $n_{A}$ (left scale) and film thickness $l$ (right scale) in the presence of saturated vapor as a function of surface polarity $\alpha$. The results obtained by TI (red circles) are compared with the droplet simulations (blue crosses) and the simulations of a water bridge (black triangles). The value for $\alpha=0.87$ lies in the complete wetting regime and is hence metastable. Inset: top view snapshots of water films (surface not shown for clarity) for three different polarities indicated on the graph. 
which corresponds to the contact angle of $\theta \simeq 134^{\circ}$ (shown on the right scale of the graph), hence reflecting high hydrophobicity. With increasing polarity $\alpha$, the dry wetting coefficient monotonically rises and finally reaches a value of around $k_{\mathrm{w}}^{\mathrm{vac}}=2.1$ for the completely polar surface $(\alpha=1)$, lying deeply in the complete wetting regime, defined by $\theta=0^{\circ}$. The wetting coefficient roughly follows the quadratic dependence $k_{\mathrm{w}}^{\mathrm{vac}}(\alpha)=a+b \alpha^{2}$, which has been attributed to dipoledipole interactions between water molecules and polar surface groups. ${ }^{38}$

\section{B. Interface potentials and the wetting coefficients}

In equilibrium, when a water droplet is deposited on a surface, it forms a microscopically thin water film, which is in equilibrium with both with the droplet and with the water vapor in its surrounding, described by a non-zero RH. Consequently, the dry wetting coefficient $k_{\mathrm{w}}^{\mathrm{vac}}$ does not exactly correspond to the equilibrium contact angle as given by Eq. (2). Instead, the "moist" wetting coefficient $k_{\mathrm{w}}$ (usually termed simply the wetting coefficient ${ }^{5}$ ) is related to the contact angle, which we evaluate in the following.

We now use the TI procedure described in Section II C to evaluate the interface potentials $\Delta f_{\mathrm{w}}\left(n_{A}\right)$ for surfaces with polarities between 0.8 and 0.87 at bulk water-vapor coexistence $(\mathrm{RH}=1)$, which are shown in Fig. 5. In all cases, the interface potential starts to decrease with the adsorbed water amount $n_{A}$. Due to the dominating entropic contribution of the first few adsorbed water molecules, we expect for small $n_{A}$ the dependence $\Delta f_{\mathrm{w}} \sim-n_{A} \log n_{A}$. For surfaces exhibiting partial wetting, the interface potential reaches a minimum at a finite $n_{A}$ and then rises again. For thicker films, it finally levels off and exhibits a plateau with the value of $1-k_{\mathrm{w}}^{\mathrm{vac}}$. The plateau corresponds to a macroscopically thick water film with two well defined interfaces: the surface-water and the watervapor interface. At the same time, the surface-vapor interface has been eliminated when the macroscopic film is formed. The free energy of the formation of a macroscopically thick film is thus

$$
\begin{aligned}
\Delta f_{\mathrm{w}}\left(n_{A} \rightarrow \infty\right) / \gamma & =\left(\gamma+\gamma_{\mathrm{sw}}-\gamma_{\mathrm{s}}\right) / \gamma \\
& =1-k_{\mathrm{w}}^{\mathrm{vac}} .
\end{aligned}
$$

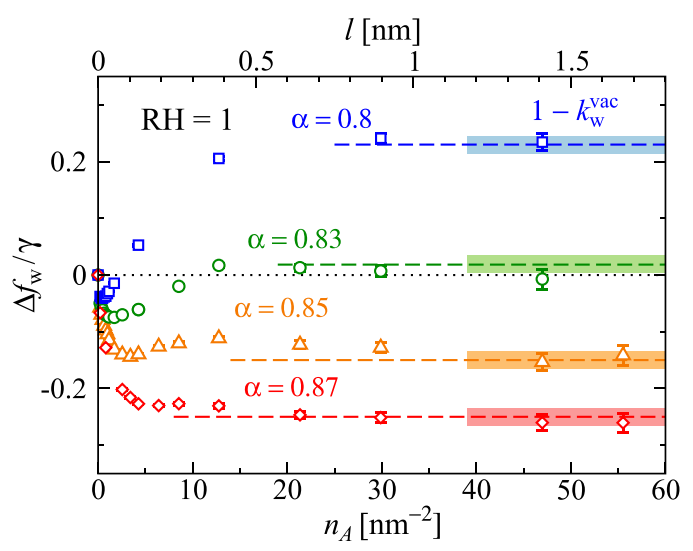

FIG. 5. Interface potentials for polar surfaces (surface polarity $\alpha$ between 0.8 and 0.87$)$ in the presence of saturated vapor $(\mathrm{RH}=1)$. The dashed lines show asymptotic values $1-k_{\mathrm{W}}^{\mathrm{vac}}$ and the thicknesses of the shaded regions represent their evaluated uncertainties $\delta k_{\mathrm{w}}^{\mathrm{vac}}$.
With an increasing polarity of the surface, the free energy minimum becomes deeper and moves toward thicker films. At polarity $\alpha=0.85$, the system displays a discontinuous wetting transition, where the microscopic film has the same free energy as the macroscopic film. This first order wetting transition is characterized by a pronounced free energy barrier, which is typical for most of the experimentally observed wetting transitions. ${ }^{37,39}$ For the polarity $\alpha=0.87$, the free energy of the plateau is already lower than the local minimum for the microscopic film, and therefore this surface polarity gives rise to the formation of a macroscopically thick water layer in saturated vapor $\mathrm{RH}=1$. The presence of a local minimum for the latter case implies the existence of a microscopically thin metastable film. ${ }^{40}$

In thermodynamic equilibrium, the actual state is the one that minimizes the free energy. Therefore, the minimum of the interface potential in Fig. 5 corresponds to the reduction of the wetting coefficient in saturated vapor relative to the scenario in vacuum

$$
\Delta k_{\mathrm{w}}=\min _{n_{A}} \Delta f_{\mathrm{w}}\left(n_{A}\right) / \gamma
$$

The reduction of the wetting coefficient is sometimes also expressed by the surface pressure $\pi_{\mathrm{sv}}=-\gamma \Delta k_{\mathrm{w}}$. ${ }^{7}$ Figure 6 shows $\Delta k_{\mathrm{w}}$ versus the corresponding film thickness for different polarities at $\mathrm{RH}=1$. The relation first follows a linear trend, which becomes non-linear for thicker films. We will discuss the reasons for this crossover in Section III E.

The wetting coefficient of a surface in contact with vapor follows as

$$
k_{\mathrm{w}}=k_{\mathrm{w}}^{\mathrm{vac}}+\Delta k_{\mathrm{w}}
$$

Using Eq. (14), we calculate the wetting coefficient for a surface in saturated vapor, which is shown by red circles in Fig. 4(a). As can be seen, the difference between the wetting coefficients $k_{\mathrm{w}}$ and $k_{\mathrm{w}}^{\mathrm{vac}}$ is insignificant for polarities below $\alpha=0.7$ (contact angles $\theta>70^{\circ}$ ) but becomes substantial as the system approaches the wetting transition at $\alpha=0.85$. When the dry wetting coefficient reaches the value $k_{\mathrm{w}}^{\mathrm{vac}}=1$, the "moist" wetting coefficient has a value $k_{\mathrm{w}} \approx 0.9$, which corresponds to the contact angle $\theta \approx 25^{\circ}$. The formation of a

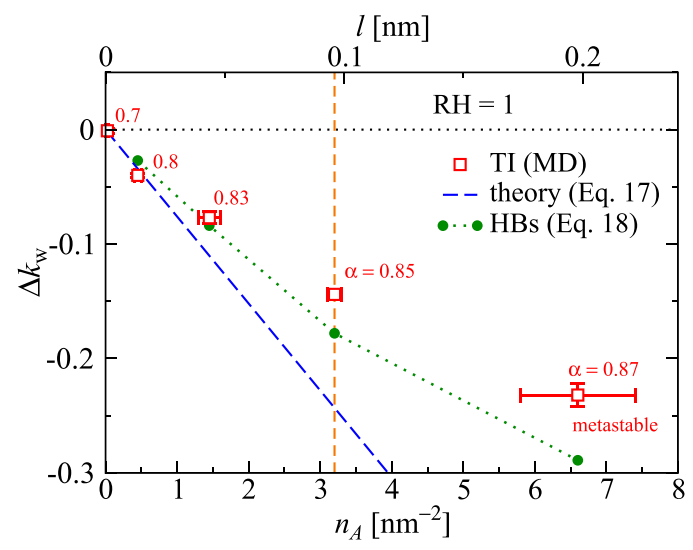

FIG. 6. Relation between the reduction of the wetting coefficient $\Delta k_{\mathrm{W}}$ (determined by the minimum of $\Delta f_{\mathrm{w}}\left(n_{A}\right)$ in Fig. 5) and the corresponding thickness of the equilibrium water film in saturated vapor (expressed as area density $n_{A}$ on the bottom scale and thickness $l$ on the top scale). The theoretical prediction, Eq. (17), is shown by a blue dashed line. The prediction based on HBs, Eq. (18), is shown by green circles connected by dotted lines. The orange dashed vertical line denotes the wetting transition. 
water film thus plays a significant role and has to be taken into account when determining the contact angle. Above the transition polarity $\alpha=0.85$, the wetting coefficient remains at unity, $k_{\mathrm{w}}=1$, since in this case the surface is completely covered with a thick water film. The discontinuity in the first derivative in $k_{\mathrm{w}}$ at the transition indicates that the wetting transition is of first order.

The water film in Fig. 4(b) is characterized by the area density, $n_{A}$, (left scale) and by the film thickness, $l=n_{A} / \rho_{\mathrm{W}}$, (right scale). The adsorbed amount gradually increases as the polarity approaches the wetting transition. The formed microscopic film has always sub-monolayer thickness $(l<0.1 \mathrm{~nm})$. The inset shows top-view snapshots of the film (surface not shown), which reveals a non-uniform distribution of water molecules in the film. Above the wetting transition $(\alpha>0.85)$, the microscopically thin film becomes metastable, and in thermodynamic equilibrium, the system gives rise to the formation of a macroscopically thick water layer.

\section{Validation of the results}

We validate the results obtained with our TI procedure by comparison with alternative simulation approaches as we explain in the following.

\section{Unrestrained droplet}

Direct evaluation of the wetting coefficient and the liquid film thickness at $\mathrm{RH} \approx 1$ for surfaces in the partial wetting regime is possible by simulations of a water droplet on the surface. By imposing cylindrical geometry of the droplet (see Fig. 7(a)) rather than simulating a droplet of spherical-cap shape, we eliminate finite-size effects due to a finite line tension. ${ }^{41-44}$ To that end, we create a large droplet composed of $N=7900$ water molecules that is periodically connected with itself via periodic boundary conditions across the shortest box size of $L_{y}=5.2 \mathrm{~nm}$ (see Fig. 7(a)). The simulation box length in this case is $L_{x}=18 \mathrm{~nm}$ for polarities $\alpha<0.7$ and $L_{x}$ $=36 \mathrm{~nm}$ for $\alpha \geq 0.7$. In the latter cases, a large surface size is needed since droplets spread substantially on polar surfaces. The simulations are around $500 \mathrm{~ns}$ long, where the first $50 \mathrm{~ns}$ are disregarded from the analysis to allow for equilibration.

We define effective boundaries of the droplet as the Gibbs dividing surfaces of water-vapor as well as water-surface interfaces. By fitting a circle to the averaged cross section of the
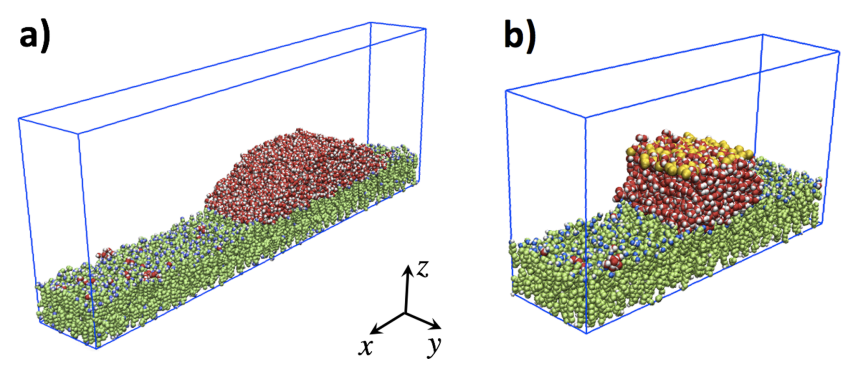

FIG. 7. Simulation snapshots of cylindrical water droplets. (a) Unrestrained cylindrical droplet replicated in the $y$-direction via periodic boundary conditions on a surface with polarity $\alpha=0.8$. (b) Water bridge (restrained droplet) spanning between the surface and 78 constrained water molecules (shown in yellow) in a plane. The water bridge serves as a bulk reservoir for the formation of a water film. water-vapor interface of the cylindrical droplet, we determine the contact angle $\theta$ of the circle with the surface. ${ }^{43,45,46}$

Since the surface of the droplet is curved, characterized by a radius of curvature $R$, the chemical potential is slightly larger than in the case of a flat water-vapor interface. The RH of the vapor above the curved surface can be calculated by Kelvin's equation for a cylindrical surface ${ }^{5,38,47}$

$$
\mathrm{RH}=\exp \left(\frac{\gamma}{k_{\mathrm{B}} T \rho_{\mathrm{w}} R}\right) .
$$

Here, $\rho_{\mathrm{w}}$ is the bulk water density and $\gamma$ corresponds strictly speaking to the water-vapor surface tension of the curved droplet interface, which differs by a factor of $1+\delta_{\mathrm{T}} / R$ from the surface tension of the flat interface. Reported values for the Tolman length $\delta_{\mathrm{T}}$ range between -0.1 and $0.1 \mathrm{~nm}^{48-50}$ Since the radii of curvature of the simulated droplets are around $R \approx 10 \mathrm{~nm}$, the curvature correction accounts for less than a factor of 0.01 . Therefore, we can safely use the value of the flat water-vapor interface, $\gamma=55 \mathrm{mN} / \mathrm{m}$, which we obtain by an independent simulation of a water slab in vapor from the diagonal pressure tensor components. ${ }^{51}$ Using Eq. (15) with $R=10 \mathrm{~nm}$, we obtain $\mathrm{RH} \approx 1.04$, which is close to saturated-vapor conditions.

The blue crosses in Fig. 4(a) (see in particular the inset) represent the wetting coefficients evaluated from the cylindrical droplet contact angle as $k_{\mathrm{w}}=\cos \theta$. The agreement between the TI and the droplet results is excellent. For polarities $\alpha>0.83$, the droplet spreads substantially on the surface and gradually transforms into a water film. Consequently, resolving its contact angle becomes difficult. The thickness of the microscopic film in such cases can still be simply estimated by restraining the droplet, as we discuss next.

\section{Water bridge (restrained droplet)}

A simple and robust approach to estimate the thickness of an adsorbed water film in cases of very low contact angles is to restrain a droplet between two interfaces in a region of the simulation box. The resulting water bridge in this case serves as a water reservoir that ensures a fixed chemical potential and enables the formation of a liquid film on the surface area not covered by the water bridge. ${ }^{52}$

We restrain the droplet by constraining the positions of 78 water molecules (shown in yellow in Fig. 7(b)) located in a plane on top of the droplet. The droplet is hence "captured" between the surface and the constrained molecules. The height of the constrained molecules is chosen such that the watervapor interfaces on both sides of the droplet are roughly flat (with only small curvature), which ensures nearly saturatedvapor conditions, $\mathrm{RH} \approx 1$, according to Eq. (15).

The simulations are around $200 \mathrm{~ns}$ long, where the first $50 \mathrm{~ns}$ are discarded to allow the system to equilibrate. We then measure the density of the water film $n_{A}$ that forms a certain distance away from the restrained water bridge.

In Fig. 4(b), we compare the thickness of the film evaluated by the TI method (red circles) with the thickness formed at the droplet (blue crosses) and at the water bridge (black triangles). All three methods yield very similar results, which validates the TI procedure. 


\section{Wetting off coexistence}

So far, we considered surfaces under two special conditions, namely, in a completely dry atmosphere or in vacuum $(\mathrm{RH}=0)$ and in saturated vapor $(\mathrm{RH}=1)$. In practical cases, however, surfaces are exposed to a vapor that is off coexistence, having an intermediate RH. In the following, we analyze and discuss how wetting properties change as a function of the RH.

The interface potentials $\Delta f_{\mathrm{w}}$ shown in Fig. 5 all correspond to the case of bulk water-vapor coexistence $(\mathrm{RH}=1)$. Evaluating the interface potential for a particular RH simply requires to replace $\rho_{\mathrm{v}}^{\text {sat }}$ by $\rho_{\mathrm{v}}=\mathrm{RH} \rho_{\mathrm{v}}^{\text {sat }}$ in Eq. (6), which leads to the expression for the interface potential for given $\mathrm{RH}$

$$
\Delta f_{\mathrm{w}}^{\mathrm{RH}}\left(n_{A}\right)=\Delta f_{\mathrm{w}}\left(n_{A}\right)-\left(k_{\mathrm{B}} T \log \mathrm{RH}\right) n_{A} .
$$

Here, $\Delta f_{\mathrm{w}}^{\mathrm{RH}}$ corresponds to the interface potential for arbitrary $\mathrm{RH}$, whereas $\Delta f_{\mathrm{w}}$ corresponds to the case of $\mathrm{RH}=1$. By knowing the interface potential for saturated vapor, we can obtain the interface potential for arbitrary RH simply by adding the term linear in $n_{A}$ in Eq. (16). The expression in the parentheses is the chemical potential shift from coexistence and defines the asymptotic behavior of the interface potential.

For the case $\alpha=0.87$, which exhibits complete wetting at coexistence, we demonstrate the influence of a finite $\mathrm{RH}$ on the interface potential in Fig. 8(a). The scenarios off coexistence ( $\mathrm{RH} \neq 1$, blue curves) are obtained by Eq. (16). In the case of undersaturated vapor $(\mathrm{RH}<1)$, the interface potentials reach a constant positive slope at large $n_{A}$ and a global minimum in the region of finite film thickness always exists. With decreasing $\mathrm{RH}$ the film thickness as well as the wetting coefficient reduction $\Delta k_{\mathrm{w}}$ shifts toward smaller values. For the limiting case $\mathrm{RH}$ $\rightarrow 0$, the slope of the second term in Eq. (16) diverges, which corresponds to the vacuum case, $n_{A}=0$ and $\Delta k_{\mathrm{w}}=0$. On the other hand, for the case of supersaturated vapor $(\mathrm{RH}>1)$, the interface potential exhibits negative slope, which implies an unlimited growth of the water film.

The presence of the free energy barrier (clearly seen in the inset of Fig. 8(a)), which separates a microscopic film from a thicker film, is typical for first order phase transitions. In order to determine the free energy minima, we fit the red data points (for RH =1) around the first minimum (for $n_{A}=0-10 \mathrm{~nm}^{-2}$ ) by $a_{0} n_{A} \log n_{A}+a_{1} n_{A}+\cdots+a_{4} n_{A}^{4}$ and the remaining data points (for $n_{A}>10 \mathrm{~nm}^{-2}$ ) by $1-k_{\mathrm{w}}^{\mathrm{vac}}+b_{2} / n_{A}^{2}+b_{3} / n_{A}^{3}$. The latter asymptotic behavior is typical for liquids with nonretarded van der Waals forces. ${ }^{53,54}$ The fits are shown by red dashed curves in the inset. The blue dashed curves are interface potentials off coexistence obtained from the fits. The equilibrium film thickness obtained as the minimum of the fits are shown by a red solid curve in Fig. 8(b) as a function of RH. Below coexistence, the water film is microscopically thin. At around $\mathrm{RH}=0.988$ (see also inset of Fig. 8(a)), the prewetting transition occurs and the system jumps from a thin sub-monolayer water film to a much thicker film composed of around two layers, $\left(n_{A} \approx 24 \mathrm{~nm}^{-2}\right.$ or $\left.l \approx 0.7 \mathrm{~nm}\right)$, which is also known as the prewetting film. ${ }^{37,55,56}$ The resulting thick prewetting film undergoes a smooth and continuous divergence toward a macroscopic film upon approaching coexistence,
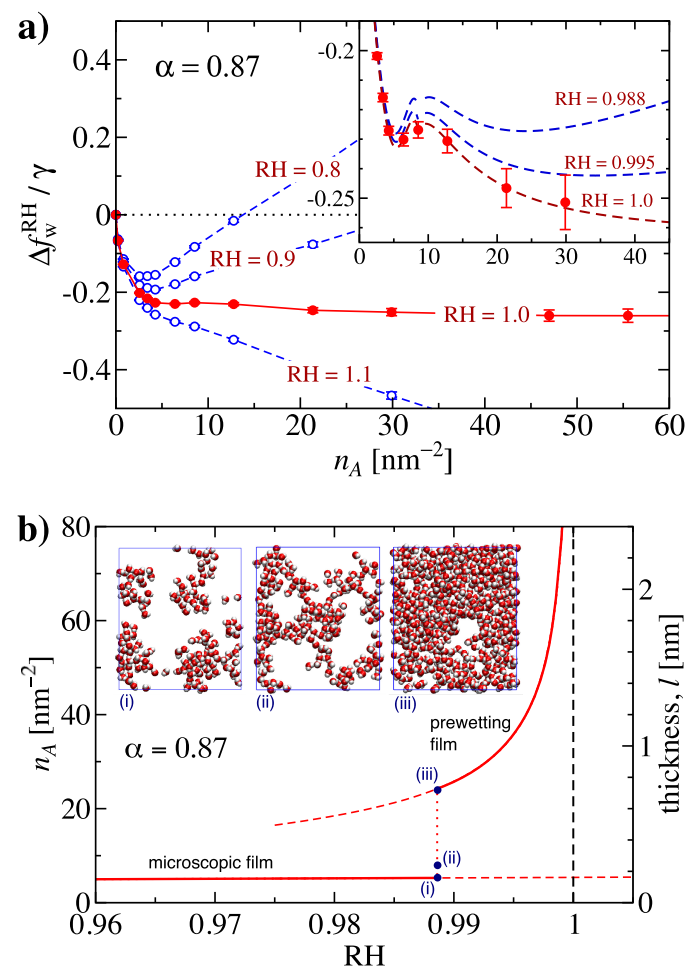

FIG. 8. (a) Interface potentials for different relative humidities (RHs) for the surface with polarity $\alpha=0.85$. Inset: enlarged region around the free energy barrier. The red dashed curve is a segmental fit to the data points at coexistence and the blue curves are corresponding interface potentials for different RHs obtained via Eq. (16). (b) Equilibrium area density (thickness on right scale) of the adsorbed water film (red solid curve) as a function of RH on the surface with polarity $\alpha=0.87$. Dashed curves show metastable states of the film. The vertical dotted line corresponds to the transient path along the prewetting transition. Inset: snapshots of water films at the prewetting transition at RH $=0.988$ for three marked thicknesses: (i) thin film, $n_{A}=5.4 \mathrm{~nm}^{-2}$, (ii) transient unstable equilibrium, $n_{A}=8.5 \mathrm{~nm}^{-2}$, and (iii) thick film, $n_{A}=24 \mathrm{~nm}^{-2}$.

$\mathrm{RH} \rightarrow 1 . .^{57,58}$ The inset in Fig. 8(b) shows liquid film snapshots at the prewetting transition (denoted along the dotted vertical transition line). As seen, the thin film prior to the transition (i) exhibits individual isolated water patches. A transient state with the area density of $n_{A}=8.6 \mathrm{~nm}^{-2}$ (corresponding to the peak of the free energy barrier) shown in (ii) has a percolated structure with inter-connected water clusters. In this case, the three-phase contact line is long and therefore line tension effects are expected to play an important role. In fact, the free energy barrier of the interface potential has been related to a positive value of the line tension. ${ }^{54}$ The stable $\sim 0.7 \mathrm{~nm}$ thick prewetting film after the transition (iii) is composed of a nearly homogeneous water double layer. The prewetting transition has been first predicted theoretically ${ }^{55,59}$ and much later observed experimentally for helium- 4 on a cesium substrate. ${ }^{60}$ It has also been a matter of intense computer modeling, mainly using monoatomic gasses ${ }^{20,61-63}$ and later also water. $^{64-66}$

The red dashed lines in Fig. 8(b) show extensions of the thick and thin film thickness into their respective metastable states off equilibrium. Metastable states accompanied by strong hysteresis due to nucleation free energy barriers ${ }^{67,68}$ are one of the most significant consequences of the firstorder nature of wetting and prewetting transitions observed experimentally. ${ }^{40,69}$ Another manifestation of the first-order 


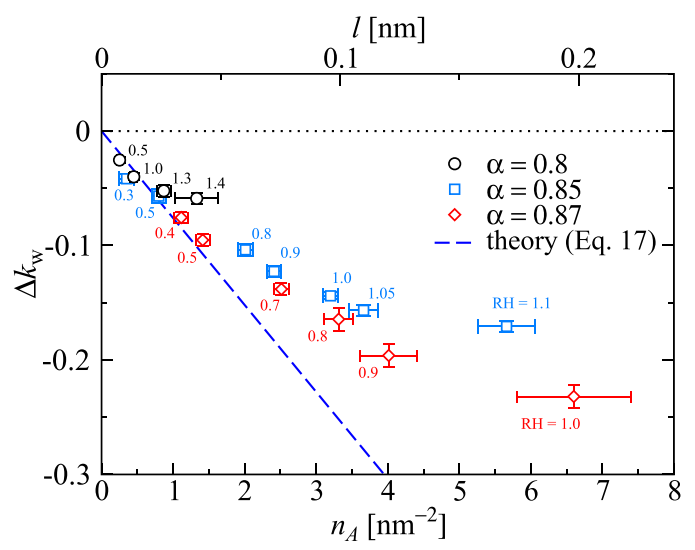

FIG. 9. Reduction of the wetting coefficient versus the thickness of adsorbed microscopic film for various RHs (denoted at each data point) for three different polarities $\alpha$. The blue dashed line shows the prediction of Eq. (17).

wetting transition is the contact angle hysteresis, mainly dominated by surface roughness and chemical heterogeneity, but on flat homogeneous surfaces also a direct consequence of the first-order nature of wetting transitions. ${ }^{37,70-72}$

Figure 9 shows the reduction of the wetting coefficient versus the film thickness at different RHs for three surface polarities. Higher RHs lead to thicker water films as well as smaller wetting coefficient $k_{\mathrm{w}}$ of the surface. We will comment on the observed trends in the following.

\section{E. Scaling predictions for the adsorbed film thickness}

We give some simple arguments for the relation between the adsorbed film density $n_{A}$ and the reduction of the wetting coefficient $\Delta k_{\mathrm{w}}$, as shown in Figs. 6 and 9. With increasing polarity, the film thickness as well as the wetting coefficient reduction monotonically grow. The relation between both can be estimated using simple thermodynamic principles. According to the Gibbs adsorption isotherm, the change in the surface-vapor surface tension is $\mathrm{d} \gamma_{\mathrm{sv}}=-\Gamma \mathrm{d} \mu$, where the surface excess $\Gamma=n_{A}$ corresponds to the adsorbed number density of molecules and $\mu$ is the vapor chemical potential. ${ }^{7}$ If the vapor density $\rho_{\mathrm{v}}$ is small enough, the adsorbed film thickness is proportional to the vapor density, $n_{A} \propto \rho_{\mathrm{v}}$. Treating the water vapor as an ideal gas, its chemical potential is $\mu=k_{\mathrm{B}} T \log \rho_{\mathrm{v}}$, which leads to $\mathrm{d} \gamma_{\mathrm{sv}}=k_{\mathrm{B}} T \mathrm{~d} n_{A}$. Expressing the change of the surface tension in terms of the wetting coefficient, $\mathrm{d} k_{\mathrm{w}}=\mathrm{d} \gamma_{\mathrm{sv}} / \gamma$, gives a linear relationship ${ }^{1}$

$$
\Delta k_{\mathrm{w}}=-\left(\frac{k_{\mathrm{B}} T}{\gamma}\right) n_{A} .
$$

This prediction is indicated as a blue dashed line in Figs. 6 and 9. As can be seen, for small thickness of the film, the prediction Eq. (17) matches the simulated values very well. For larger film thickness, the wetting coefficient reduction is smaller than predicted by the theory. The span of the linear regime depends also on the polarity of the surface. From Fig. 9, it follows that for more polar surfaces, the linear regime extends to thicker films, whereas for lower polarities, the data points deviate from the linear prediction already for thinner films.

The theoretical prediction given by Eq. (17) is based on the assumption that the adsorbed film thickness is proportional to the water vapor density, which is the case as long as the adsorbed water molecules do not significantly interact with each other. The inset in Fig. 4(b) shows snapshots of the equilibrium sub-monolayer films formed on surfaces of different polarities at $\mathrm{RH}=1$. As is clearly evident, the water molecules are not uniformly distributed on the surfaces but rather tend to aggregate into flat clusters. Similar faceted islands of water have been observed also experimentally on various flat surfaces, ${ }^{73,74}$ which indicates that water molecules strongly interact with each other in a film.

In order to qualitatively explain the observed trends for thicker films, we apply the concepts of the virial expansion. Figure 10(a) shows the lateral radial distribution function (RDF), $g_{2 \mathrm{D}}(r)$, between water oxygen atoms in the infinite dilution limit, realized by simulating two water molecules on the surface. The lateral RDF in the infinite dilution limit allows us to compute the second surface virial coefficient as $B_{2}^{(2 \mathrm{D})}=-\pi \int_{0}^{\infty}\left[g_{2 \mathrm{D}}(r)-1\right] r \mathrm{~d} r$. The resulting virial coefficients $B_{2}^{(2 D)}$ are plotted in Fig. 10(b) as a function of surface polarity $\alpha$. The high peaks in the RDFs and the negative values of the virial coefficients imply strong attraction between water molecules. However, with increasing polarity, the effective affinity between water molecules is reduced. This effect can be easily understood in terms of hydrogen bonds (HBs) that water molecules form. Ideally, a water molecule can form maximally four HBs with surrounding molecules. The water molecules in the adsorbed film spend this HB capacity partly to form HBs with the surface and partly to form HBs with other water molecules in the film. The more polar the surface, the more HBs a water molecule
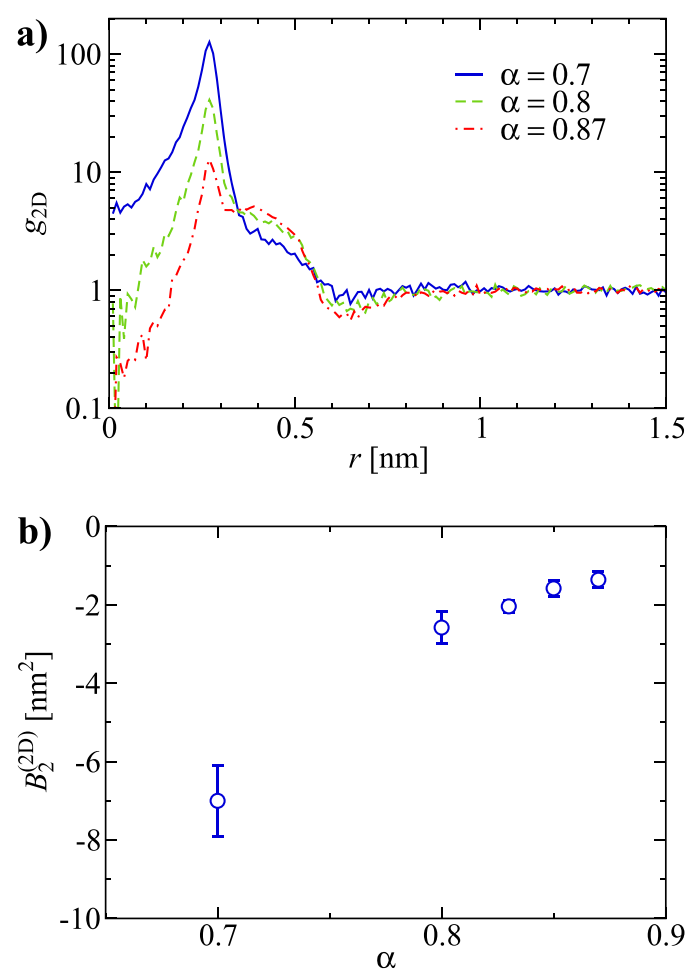

FIG. 10. (a) Log-linear plot of the lateral radial distribution function $g_{2 \mathrm{D}}(r)$ between the water oxygens in the infinite dilution limit on surfaces with different polarity $\alpha$. (b) Second surface virial coefficient $B_{2}^{(2 D)}$ as a function of the surface polarity $\alpha$. 
tends to form with the surface head groups, and thereby the tendency to hydrogen bond with other water molecules is reduced.

As we show in the supplementary material, the secondorder virial expansion of the free energy yields the correction factor of $1+B_{2}^{(2 D)} n_{A}$ to the linear expression in Eq. (17). Since in our case $B_{2}^{(2 \mathrm{D})}<0$ (i.e., water molecules attract each other), the correction decreases the effect of water film on the wetting coefficient reduction $\Delta k_{\mathrm{w}}\left(n_{A}\right)$. However, the virial expansion is justified only when the second-order correction is significantly smaller than the leading order. For the considered cases with $n_{A}>1 \mathrm{~nm}^{-2}$, the correction is $\left|B_{2}^{(2 \mathrm{D})} n_{A}\right|>1$; therefore, the virial expansion breaks down and we cannot use it for quantitative predictions. Nevertheless, the virial-expansion approach offers a qualitative explanation for the observed nonlinear dependence of the wetting coefficient reduction with increasing film thickness in Figs. 6 and 9.

Alternatively, the wetting film properties can be discussed in terms of the number of hydrogen bonds formed between water and surface molecules. Equation (17) implies that the surface free energy is lowered by $k_{\mathrm{B}} T$ for each adsorbed water molecule in the film. In thicker films, not all water molecules participate in the binding with the surface, as opposed to cases of thin films, where isolated water molecules on the surface entirely participate in HB formation. Consequently, the reduction of the wetting coefficient is smaller than predicted by Eq. (17). We perform a simple analysis by counting the number of adsorbed water molecules $N_{\text {ads }}^{\mathrm{HB}}$ that form at least one $\mathrm{HB}$ with the surface. We use the concept of HBs since it is a well established tool to determine the binding strength of hydrated systems. We determine $\mathrm{HBs}$ from the simulation trajectories by employing the widely used Luzar-Chandler geometric criterion. ${ }^{75}$ The resulting $\mathrm{HB}$ area density $n_{A}^{\mathrm{HB}}=N_{\text {ads }}^{\mathrm{HB}} / A$ represents an "effective" area density of the water film that is directly in contact with the surface. Note that $N_{\text {ads }}^{\mathrm{HB}} \leq N_{\text {ads }}$ since not all of the $N_{\text {ads }}$ adsorbed water molecules necessarily form HBs with the surface and consequently $n_{A}^{\mathrm{HB}} \leq n_{A}$. The value $n_{A}^{\mathrm{HB}}$ replaces the total film area density $n_{A}$ in Eq. (17), which gives an estimation for the wetting coefficient reduction as

$$
\Delta k_{\mathrm{w}} \approx-\left(\frac{k_{\mathrm{B}} T}{\gamma}\right) n_{A}^{\mathrm{HB}} .
$$

The prediction of this equation is shown by green data points in Fig. 6. It clearly gives better predictions than Eq. (17) (blue dashed line). However, Eq. (18) still slightly overestimates the value of $\Delta k_{\mathrm{w}}$ because the molecules that participate in hydrogen bonding with the surface can partially participate also in water-water binding, therefore making the surface-water interactions slightly weaker.

Clearly, the observed behavior is not necessarily restricted to water but may be relevant also for other liquids that do not form HBs. However, some features, such as the dependence of the second virial coefficient on the surface polarity (Fig. 10) and the induced larger deviations from the linear wetting predictions on less polar surfaces (Fig. 9), have to do with the specific nature of hydrogen bonding, where the saturation property of $\mathrm{HBs}$ leads to a competition between water-water and surface-water HBs.

\section{CONCLUSIONS}

In this study, we employ Molecular Dynamics simulations to investigate wetting properties of a model surface in contact with water vapor. The surface is composed of alkane chains terminated by head groups of controlled degree of polarity. We demonstrate how a modified thermodynamic integration (TI) procedure, implemented in most popular simulation packages, can be used to determine the interface wetting potential of a surface, that is, the change of the surface free energy as a function of the adsorbed water film thickness at given humidity conditions with a very high degree of accuracy.

Increasing the surface polarity increases the hydrophilicity of the surface, which leads to an increased wetting coefficient and decreased contact angle. In the presence of water vapor, a quasi two-dimensional sub-monolayer water film forms on the surface, whose thickness depends on the surface polarity and the relative humidity. The formation of the film lowers the interfacial surface energy and consequently increases the contact angle of the surface. The extent and effect of the water film is most pronounced for polar surfaces near the wetting transition. We validate the results obtained by the TI procedure by performing additional simulations of water droplets and water bridges. We find excellent agreement between the wetting coefficients and the water film thicknesses obtained from both approaches.

For highly polar surfaces, we also locate the prewetting transition, which occurs slightly below the water-vapor coexistence and is characterized by a jump of the layer thickness from a sub-monolayer film to a film composed of two water layers.

For cases when the formed film is thin, the reduction of the surface wetting coefficient is linearly proportional to the film thickness, as can be deduced from simple thermodynamic assumptions. Yet for thicker films, which form at higher RHs or more polar surfaces, the linear dependence breaks down. The deviations from linearity can be qualitatively explained by clustering of the molecules due to HBs. Since on less polar surfaces the water molecules form more HBs among themselves rather than fully participating in the binding to the surface, the linear regime breaks down earlier on less polar surfaces compared with more polar surfaces.

\section{SUPPLEMENTARY MATERIAL}

See supplementary material for the virial expansion correction to Eq. (17).

\section{ACKNOWLEDGMENTS}

Funding by the Deutsche Forschungsgemeinschaft (DFG) via Grant No. NE 810/10 is gratefully acknowledged.

${ }^{1}$ D. Bonn, J. Eggers, J. Indekeu, J. Meunier, and E. Rolley, Rev. Mod. Phys. 81, 739 (2009).

${ }^{2}$ R. Seemann, M. Brinkmann, E. J. Kramer, F. F. Lange, and R. Lipowsky, Proc. Natl. Acad. Sci. U. S. A. 102, 1848 (2005).

${ }^{3}$ A. Méndez-Vilas, A. B. Jódar-Reyes, and M. L. González-Martín, Small 5, 1366 (2009)

${ }^{4}$ A. Rosenhahn, S. Schilp, H. J. Kreuzer, and M. Grunze, Phys. Chem. Chem. Phys. 12, 4275 (2010). 
${ }^{5}$ J. S. Rowlinson and B. Widom, Molecular Theory of Capillarity (Dover Publications, New York, 2002).

${ }^{6}$ P. G. de Gennes, Rev. Mod. Phys. 57, 827 (1985).

${ }^{7}$ L. J. Schlangen, L. K. Koopal, M. A. C. Stuart, and J. Lyklema, Colloids Surf., A 89, 157 (1994).

${ }^{8}$ E. M. Grzelak and J. R. Errington, J. Chem. Phys. 128, 014710 (2008).

${ }^{9}$ D. Bratko, R. A. Curtis, H. W. Blanch, and J. M. Prausnitz, J. Chem. Phys. 115, 3873 (2001).

${ }^{10}$ K. Leung, A. Luzar, and D. Bratko, Phys. Rev. Lett. 90, 065502 (2003).

${ }^{11}$ X. Huang, C. J. Margulis, and B. J. Berne, Proc. Natl. Acad. Sci. U. S. A. 100, 11953 (2003).

${ }^{12}$ N. Choudhury and B. M. Pettitt, J. Phys. Chem. B 110, 8459 (2006).

${ }^{13}$ A. J. Patel, P. Varilly, and D. Chandler, J. Phys. Chem. B 114, 1632 (2010).

${ }^{14}$ A. J. Patel, P. Varilly, S. N. Jamadagni, H. Acharya, S. Garde, and D. Chandler, Proc. Natl. Acad. Sci. U. S. A. 108, 17678 (2011).

${ }^{15}$ M. Kanduč, A. Schlaich, E. Schneck, and R. R. Netz, Langmuir 32, 8767 (2016).

${ }^{16}$ G. J. Gloor, G. Jackson, F. J. Blas, and E. de Miguel, J. Chem. Phys. 123, 134703 (2005).

${ }^{17}$ F. Leroy, D. J. V. A. dos Santos, and F. Müller-Plathe, Macromol. Rapid Commun. 30, 864 (2009).

${ }^{18}$ M. Kanduč, E. Schneck, and R. R. Netz, Chem. Phys. Lett. 610-611, 375 (2014).

${ }^{19}$ F. Leroy and F. Müller-Plathe, Langmuir 31, 8335 (2015).

${ }^{20}$ J. R. Errington, Langmuir 20, 3798 (2004).

${ }^{21}$ K. S. Rane, V. Kumar, and J. R. Errington, J. Chem. Phys. 135, 234102 (2011).

${ }^{22}$ V. Kumar and J. R. Errington, Mol. Simul. 39, 1143 (2013).

${ }^{23}$ V. Kumar and J. R. Errington, J. Phys. Chem. C 117, 23017 (2013).

${ }^{24}$ M. Kanduč and R. R. Netz, Proc. Natl. Acad. Sci. U. S. A. 112, 12338 (2015).

${ }^{25}$ C. Oostenbrink, A. Villa, A. E. Mark, and W. F. Van Gunsteren, J. Comput. Chem. 25, 1656 (2004).

${ }^{26}$ H. J. C. Berendsen, J. R. Grigera, and T. P. Straatsma, J. Phys. Chem. 91, 6269 (1987).

${ }^{27}$ D. Van Der Spoel, E. Lindahl, B. Hess, G. Groenhof, A. E. Mark, and H. J. C. Berendsen, J. Comput. Chem. 26, 1701 (2005).

${ }^{28}$ S. Pronk, S. Páll, R. Schulz, P. Larsson, P. Bjelkmar, R. Apostolov, M. R. Shirts, J. C. Smith, P. M. Kasson, D. van der Spoel, B. Hess, and E. Lindahl, Bioinformatics 29, 845 (2013).

${ }^{29}$ H. J. C. Berendsen, J. P. M. Postma, W. F. van Gunsteren, A. DiNola, and J. R. Haak, J. Chem. Phys. 81, 3684 (1984).

${ }^{30}$ T. Darden, D. York, and L. Pedersen, J. Chem. Phys. 98, 10089 (1993).

${ }^{31}$ U. Essmann, L. Perera, M. L. Berkowitz, T. Darden, H. Lee, and L. G. Pedersen, J. Chem. Phys. 103, 8577 (1995).

${ }^{32}$ K. Lum, D. Chandler, and J. D. Weeks, J. Phys. Chem. B 103, 4570 (1999).

${ }^{33}$ J. R. Errington and A. Z. Panagiotopoulos, J. Phys. Chem. B 102, 7470 (1998).

${ }^{34}$ D. Frenkel and A. J. Ladd, J. Chem. Phys. 81, 3188 (1984).

${ }^{35}$ T. C. Beutler, A. E. Mark, R. C. van Schaik, P. R. Gerber, and W. F. van Gunsteren, Chem. Phys. Lett. 222, 529 (1994).

${ }^{36}$ S. Dietrich and M. Napiórkowski, Phys. Rev. A 43, 1861 (1991).
${ }^{37}$ D. Bonn and D. Ross, Rep. Prog. Phys. 64, 1085 (2001).

${ }^{38}$ N. Giovambattista, A. B. Almeida, A. M. Alencar, and S. V. Buldyrev, J. Phys. Chem. C 120, 1597 (2016).

${ }^{39}$ S. R. Friedman, M. Khalil, and P. Taborek, Phys. Rev. Lett. 111, 226101 (2013).

${ }^{40}$ D. Bonn, H. Kellay, and G. Wegdam, Phys. Rev. Lett. 69, 1975 (1992).

${ }^{41}$ T. Getta and S. Dietrich, Phys. Rev. E 57, 655 (1998).

${ }^{42}$ T. Werder, J. H. Walther, R. L. Jaffe, T. Halicioglu, and P. Koumoutsakos, J. Phys. Chem. B 107, 1345 (2003).

${ }^{43}$ J. H. Weijs, A. Marchand, B. Andreotti, D. Lohse, and J. H. Snoeijer, Phys. Fluids 23, 022001 (2011).

${ }^{44}$ J. Zhang, F. Leroy, and F. Müller-Plathe, Phys. Rev. Lett. 113, 046101 (2014).

${ }^{45}$ H. Peng, A. V. Nguyen, and G. R. Birkett, Mol. Simul. 38, 945 (2012).

${ }^{46}$ H. Peng, G. R. Birkett, and A. V. Nguyen, Mol. Simul. 40, 934 (2014).

${ }^{47}$ M. H. Factorovich, V. Molinero, and D. A. Scherlis, J. Am. Chem. Soc. 136, 4508 (2014).

${ }^{48}$ D. M. Huang, P. L. Geissler, and D. Chandler, J. Phys. Chem. B 105, 6704 (2001).

${ }^{49}$ H. S. Ashbaugh and L. R. Pratt, Rev. Mod. Phys. 78, 159 (2006).

${ }^{50}$ F. Sedlmeier and R. R. Netz, J. Chem. Phys. 137, 135102 (2012).

${ }^{51}$ M. Nijmeijer, C. Bruin, A. Bakker, and J. Van Leeuwen, Phys. Rev. A 42, 6052 (1990).

${ }^{52}$ N. Tretyakov, M. Müller, D. Todorova, and U. Thiele, J. Chem. Phys. 138, 064905 (2013).

${ }^{53}$ M. E. Fisher, J. Chem. Soc., Faraday Trans. 2 82, 1569 (1986).

${ }^{54}$ J. Indekeu, Phys. A 183, 439 (1992).

${ }^{55}$ J. W. Cahn, J. Chem. Phys. 66, 3667 (1977).

${ }^{56}$ E. Hauge and M. Schick, Phys. Rev. B 27, 4288 (1983).

${ }^{57}$ R. Lipowsky and D. Kroll, Phys. Rev. Lett. 52, 2303 (1984).

${ }^{58}$ R. Lipowsky, Phys. Rev. B 32, 1731 (1985).

${ }^{59}$ C. Ebner and W. Saam, Phys. Rev. Lett. 38, 1486 (1977).

${ }^{60}$ P. Taborek and J. Rutledge, Phys. Rev. Lett. 68, 2184 (1992).

${ }^{61}$ J. Finn and P. Monson, Phys. Rev. A 39, 6402 (1989).

${ }^{62}$ W. Shi, X. Zhao, and J. K. Johnson, Mol. Phys. 100, 2139 (2002).

${ }^{63}$ S. Toxvaerd, J. Phys. Chem. C 111, 15620 (2007).

${ }^{64}$ I. Brovchenko, A. Geiger, and A. Oleinikova, J. Chem. Phys. 120, 1958 (2004).

${ }^{65}$ X. Zhao, Phys. Rev. B 76, 041402 (2007).

${ }^{66}$ M. Gordillo and J. Martí, Phys. Rev. E 84, 011602 (2011).

${ }^{67}$ M. Schick and P. Taborek, Phys. Rev. B 46, 7312 (1992).

${ }^{68}$ R. Bausch, R. Blossey, and M. Burschka, J. Phys. A: Math. Gen. 27, 1405 (1994).

${ }^{69}$ H. Kellay, D. Bonn, and J. Meunier, Phys. Rev. Lett. 71, 2607 (1993).

${ }^{70}$ E. Chibowski, Adv. Colloid Interface Sci. 103, 149 (2003).

${ }^{71}$ V. M. Starov and M. Velarde, J. Phys.: Condens. Matter 21, 464121 (2009).

${ }^{72}$ I. V. Kuchin and V. M. Starov, Langmuir 32, 5333 (2016).

${ }^{73}$ A. Verdaguer, G. Sacha, H. Bluhm, and M. Salmeron, Chem. Rev. 106, 1478 (2006).

${ }^{74}$ K. Xu, P. Cao, and J. R. Heath, Science 329, 1188 (2010).

${ }^{75}$ A. Luzar and D. Chandler, Nature 379, 55 (1996). 